\title{
Loss of mechanosensitive sclerostin may accelerate cranial bone growth and regeneration
}

\author{
Kyung Shin Kang, PhD, ${ }^{1,2}$ Jeff Lastfogel, MD, ${ }^{3}$ Laurie L. Ackerman, MD, ${ }^{4}$ Andrew Jea, MD, ${ }^{4}$ \\ Alexander G. Robling, PhD, ${ }^{1,2,5}$ and Sunil S. Tholpady, MD, PhD',3 \\ Departments of ${ }^{1}$ Anatomy \& Cell Biology, ${ }^{3}$ Surgery, and ${ }^{4}$ Neurosurgery, Indiana University School of Medicine, Indianapolis; \\ ${ }^{2}$ Richard L. Roudebush VA Medical Center, Indianapolis; and ${ }^{5}$ Department of Biomedical Engineering, Indiana University-Purdue \\ University at Indianapolis, Indiana
}

\begin{abstract}
OBJECTIVE Cranial defects can result from trauma, infection, congenital malformations, and iatrogenic causes and represent a surgical challenge. The current standard of care is cranioplasty, with either autologous or allogeneic material. In either case, the intrinsic vascularity of the surrounding tissues allows for bone healing. The objective of this study was to determine if mechanotransductive gene manipulation would yield non-weight-bearing bone regeneration in a critical size calvarial defect in mice.
\end{abstract}

\begin{abstract}
METHODS A mouse model of Sost deletion in Sost knockout (KO) mice was created in which the osteocytes do not express sclerostin. A critical size calvarial defect (4 $\mathrm{mm}$ in diameter) was surgically created in the parietal bone in 8-week-old wild-type $(n=8)$ and Sost KO $(n=8)$ male mice. The defects were left undisturbed (no implant or scaffold) to simulate a traumatic calvariectomy model. Eight weeks later, the animals were examined at necropsy by planimetry, histological analysis of new bone growth, and micro-CT scanning of bone thickness.
\end{abstract}

RESULTS Defects created in wild-type mice did not fill with bone over the study period of 2 months. Genetic downregulation of sclerostin yielded animals that were able to regenerate $40 \%$ of the initial critical size defect area 8 weeks after surgery. A thin layer of bone covered a significant portion of the original defect in all Sost KO animals. A statistically significant increase in bone volume $(p<0.05)$ was measured in Sost KO mice using radiodensitometric analysis. Immunohistochemical analysis also confirmed that this bone regeneration occurred through the Wnt pathway and originated from the edge of the defect; BMP signaling did not appear to be affected by sclerostin.

CONCLUSIONS Mechanical loading is an important mechanism of bone formation in the cranial skeleton and is poorly understood. This is partially due to the fact that it is difficult to load bone in the craniomaxillofacial skeleton. This study suggests that modulation of the Wnt pathway, as is able to be done with monoclonal antibodies, is a potentially efficacious method for bone regeneration that requires further study.

https://thejns.org/doi/abs/10.3171/2017.5.JNS17219

KEY WORDS critical size defect; sclerostin; bone regeneration; cranial reconstruction; tissue engineering

$\mathrm{R}$ ECONSTRUCTION of bony defects in the cranial and facial skeleton is challenging. These defects are commonly seen as a result of pathologic processes, including congenital malformations, oncological extirpation, trauma, and infections. ${ }^{6}$ Such a clinical situation with sufficiently large defects can overwhelm the capacity of the innate healing mechanisms for repair and regeneration. This is known as a critical size defect, which is generally regarded as defect healing of less than $10 \%$ over the lifetime of an organism if left untreated. ${ }^{31}$ There are several reconstructive options to be considered, but most have limitations or risks inherent in the procedure. Autogenous bone grafts, long considered to be the gold standard in cranial and facial bony reconstruction, are limited in supply and pose surgical risks associated with a second surgical site. ${ }^{39}$ Allogenic (or cadaveric) bony tissue has limitations related to poor mechanical properties and degradability.22 Although demineralized bone matrix carries growth fac-

ABBREVIATIONS BMP = bone morphogenetic protein; $\mathrm{HRP}=$ horseradish peroxidase; $\mathrm{KO}=$ knockout; $\mathrm{WT}=$ wild type.

SUBMITTED January 24, 2017. ACCEPTED May 15, 2017.

INCLUDE WHEN CITING Published online November 10, 2017; DOI: 10.3171/2017.5.JNS17219. 
tors, such as bone morphogenetic proteins (BMPs), and is osteoinductive, a host immune response can occur despite processing, which limits its utility. ${ }^{1,49}$ Synthetic materials are avascular, and a lack of integration or exposure can lead to displacement, infection, and extrusion.?

Sclerostin, a Wnt pathway antagonist, is an emerging target molecule for bone disease. The canonical Wnt signaling pathway can be altered and enhanced with neutralizing antibodies that target sclerostin, leading to abnormal bone metabolism. ${ }^{18,28,30}$ Sclerostin is a protein product of the Sost gene and has a powerful inhibitory effect mainly on low-density lipoprotein receptor-related protein-5 (Lrp5). ${ }^{28}$ Sclerostin is the biochemical link between loading and bone formation, with sclerostin levels decreasing with increased loading and subsequent bone formation. ${ }^{26}$ Recent reports have demonstrated that sclerostin antibody therapy can be effective in treating osteoporosis and improving fracture outcomes. ${ }^{32,37}$ Successful clinical trials with sclerostin antibody therapy have been performed, and preliminary results show it to be a promising treatment for fracture prevention. ${ }^{17}$

Sclerostin-targeting treatments can be considered not only for low bone mass-related bone diseases but also for reconstruction options. However, treatment strategies for bony wound healing in the craniofacial skeleton differ from those for the skeleton below the clavicles with regard to the role that loading assists with bone formation. ${ }^{23}$ In the skeleton below the clavicles, rigid stabilization is followed by loading to enhance bone formation, structural integration, and eventual return to normal function. This is the natural process of osteocytes embedded in load-bearing bone matrix to integrate mechanical loading into bone homeostasis during modeling and remodeling. ${ }^{29}$ Meanwhile, in the cranial and most of the facial skeleton, loading is not clinically applicable or practical in fracture healing. These differences above and below the clavicle are due to structural and biochemical differences. ${ }^{2}$

Previous studies have observed that a lack of sclerostin increased parietal bone thickness in mice, suggesting that sclerostin targeting might be useful not only for the weight-bearing skeleton but also for the non-weightbearing skeleton. ${ }^{19-21,26}$ This study was designed to test the hypothesis that non-weight-bearing portions of the craniofacial skeleton were sclerostin responsive, and that downregulation of sclerostin would help heal critical size defects in mice.

\section{Methods}

\section{Critical Size Calvarial Defect Model}

We obtained approval from the Indiana University Animal Care and Use Committee for the following animal experiments. Eight male adult wild-type (WT) and Sost knockout (KO) animals were identified for calvarial defect creation. Wild-type mice were purchased from the Jackson Laboratory, and Sost KO mice were generated as previously described. ${ }^{13}$ At 8 weeks of age, all animals were anesthetized with 5\% isoflurane inspiration using a facial mask. The skin was sterilized with iodine and ethanol. An incision was made sagittally at the top of the skull, with full-thickness retraction of the skin and periosteum, to expose the calvaria. Four-millimeter critical size defects were created in the parietal bone with a trephine bur under constant saline irrigation. ${ }^{3,15,35,36}$ Once the defects were made, they were thoroughly irrigated and closed with a resorbable suture at the skin level. After surgery, carprofen was used to minimize pain or discomfort, and all animals were monitored for signs of infection and unmanaged pain.

\section{Western Blot}

To confirm sclerostin levels in the bone of Sost KO mice, protein was extracted from the femurs of 8-weekold Sost KO and WT mice. The bones were homogenized in T-Per protein extraction reagent (ThermoFisher Scientific). The centrifuged supernatant was collected for total protein. Protein concentration was determined using a BCA Protein Assay Kit (Pierce). Equal amounts of protein were separated by sodium dodecyl sulfate-polyacrylamide gel electrophoresis and transferred to polyvinylidene fluoropolymer. The membrane was blocked with $5 \%$ skim milk in TBS-T for 1 hour. The membranes were then incubated with a goat anti-sclerostin antibody (R\&D systems) and anti- $\beta$-actin antibody (Sigma) at a dilution of 1:1000 at $4^{\circ} \mathrm{C}$ overnight. The blots were incubated in horseradish peroxidase (HRP)-conjugated secondary antibodies, and immune complexes were detected using the WesternBreeze chemiluminescent kit (Life Technologies).

\section{Micro-CT Scanning}

Calvarial bone density was measured using a vivaCT 40 (Scanco Medical) scanner at 1 day, 4 weeks, and 8 weeks after surgery. Scans were obtained in isofluraneanesthetized mice in which the scanning condition was as follows: isotropic voxel size $17.5 \mu \mathrm{m}^{3}, 55 \mathrm{kVp}$, and integration time $200 \mathrm{msec}$. The relatively newly formed bone area was evaluated by comparing the values with each first scan within a manually delineated region of interest. The new bone volume formed was calculated as the bone volume at Week 8 over the bone volume on Day 1 within each group.

\section{Histopathological Analysis}

Two months after surgery, the animals were euthanized with an intracardiac injection of lidocaine. The skulls were removed en bloc and were fixed in Carnoy's fixative and decalcified with a $14 \%$ EDTA solution. They were cut in the sagittal plane, embedded in paraffin, and cut into $7-\mu \mathrm{m}$ sections. Sections were either stained with H \& E or processed for immunohistochemical analysis. For immunohistochemical staining, sections were rehydrated through a graded alcohol series. Endogenous peroxidase was blocked by being incubated for 20 minutes in an $\mathrm{MeOH} / \mathrm{H}_{2} \mathrm{O}_{2}$ solution. Slides were blocked for 30 minutes in $2 \%$ goat serum. Primary antibody at a concentration of 1:100 was incubated with the slides overnight at $4^{\circ} \mathrm{C}$. After 3 washes in phosphate-buffered saline, the slides were exposed to goat anti-mouse HRP secondary at 1:500 for 2.5 hours. The secondary was rinsed off with phosphatebuffered saline, and 3,3'-diaminobenzidine was used to localize the HRP signal. Slides were dehydrated and mounted in a xylene-based fixative. 


\section{Statistical Analysis}

Statistical analyses were computed using ANOVA for $\mathrm{WT}$ and Sost KO mice. Significance was taken at $\mathrm{p}<0.05$. All data are presented as the mean \pm SEM.

\section{Results}

\section{Sclerostin Expression in Sost KO Mice}

To determine whether sclerostin in bone could be successfully downregulated in Sost KO mice, we examined the endogenous levels of sclerostin in protein extracts from Sost $\mathrm{KO}$ and WT mice (Fig. 1A). Sclerostin was present in WT mice in all bone specimens examined. However, no immunodetectable sclerostin protein was present in Sost $\mathrm{KO}$ mice.

\section{Downregulation of Sclerostin and Bone Regeneration in the Craniofacial Skeleton}

To evaluate bone regeneration with sclerostin downregulation (biochemically mimicking loading), we used the standard mouse calvarial bone defect model. Critical size defects measuring $4 \mathrm{~mm}$ in diameter were created in both WT and Sost KO mice. Interestingly, the thickness of bone was significantly different, as reported previously (data not shown). ${ }^{21}$ The defects were otherwise made with- out issue. Upon necropsy at 8 weeks, all defects had the appearance of a thin covering of tissue. Grossly, however, Sost KO mice had more bone covering the defect. The values obtained from micro-CT analysis bore out this observation (Fig. 1B-D). More defect area was covered by bone in Sost KO mice over 8 weeks by $40 \%$ compared with the initial bone area, whereas new bone was merely formed along the defect edge in WT mice. The bone regeneration in WT mice did not statistically differ from the initial surgery time point within the same period. The calculated new bone volume shows that sclerostin downregulation contributed to accelerating new bone formation in the non-weight-bearing bone.

Histopathological analysis is shown in Fig. 2. Bone was present in the form of a growing osteogenic front at the edge of the defect 8 weeks after surgery in Sost KO mice. In contrast, WT mice demonstrated no bone formed by the dura, and there were no islands of bone present within the defect in WT mice, which are consistent with the micro$\mathrm{CT}$ data in Fig. 1. Another difference was that Sost KO mice showed gradual bone growth toward the center of the defect, whereas WT mice did not show a gradually closing bone defect and instead exhibited fibrous tissues. We observed that the newly formed bone in the Sost KO
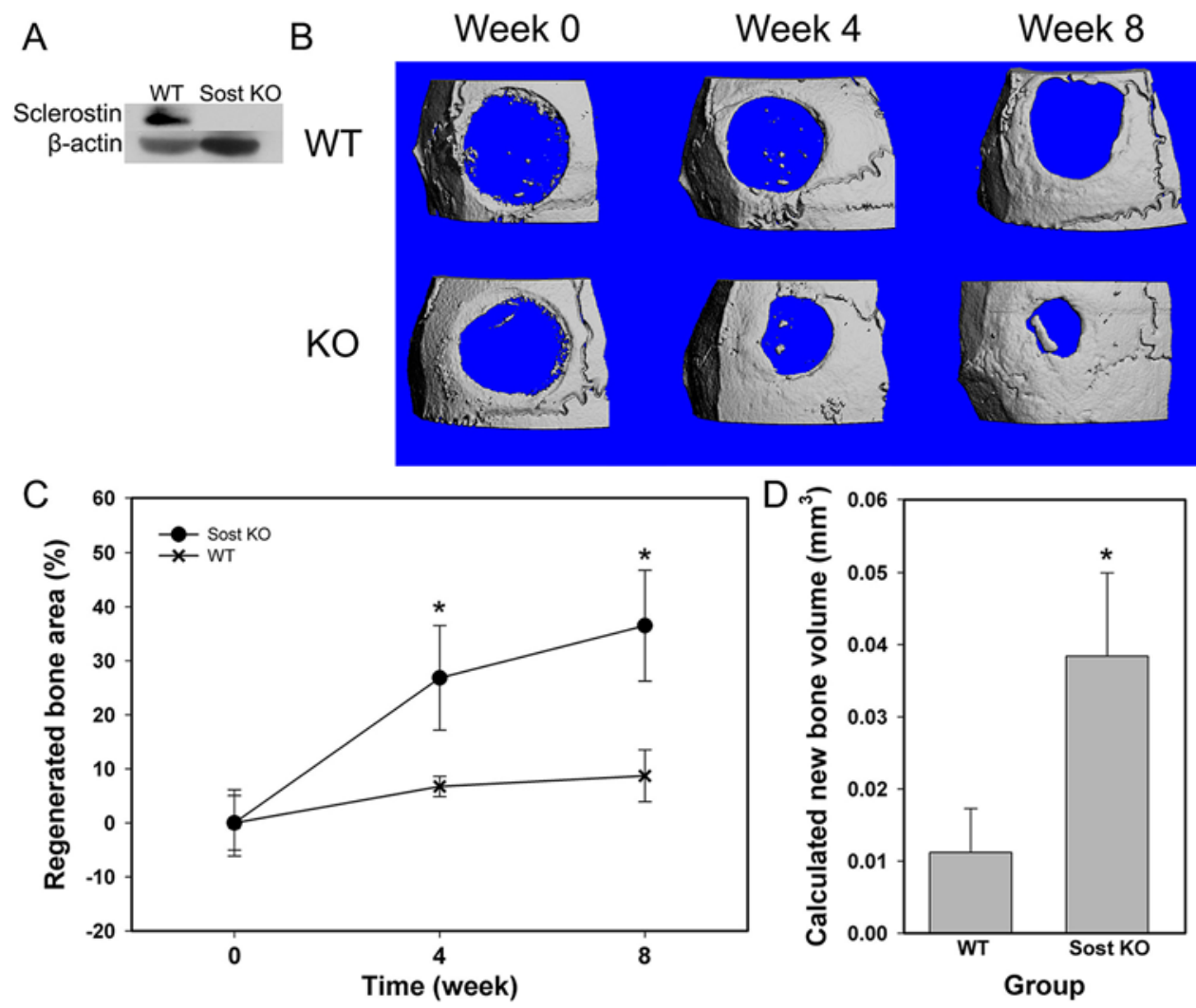

FIG. 1. A: Western blot result showing that Sost KO mice did not express sclerostin in bone, while WT mice had sclerostin in bone. B-D: Micro-CT analysis of mice at time points of postoperatively, 4 weeks, and 8 weeks. Reconstructed micro-CT images after surgery showing that Sost KO mice remarkably gained new bone compared with WT mice over time (B). The percentage of regenerated bone over time is normalized to the initial bone area of each group. There was close to $40 \%$ bone coverage over the defect 8 weeks after defect generation, while WT mice did not gain a significant amount of new bone (C). The new bone volume was calculated based on the reconstructed micro-CT images by subtracting the initial bone volume (Week 0 ) from the bone volume at Week 8 . The area of bone formed was significantly larger than that in WT animals (D). Values are mean \pm SEM. All differences are statistically significant. ${ }^{*} p<0.05$ compared with the WT group. Figure is available in color online only. 

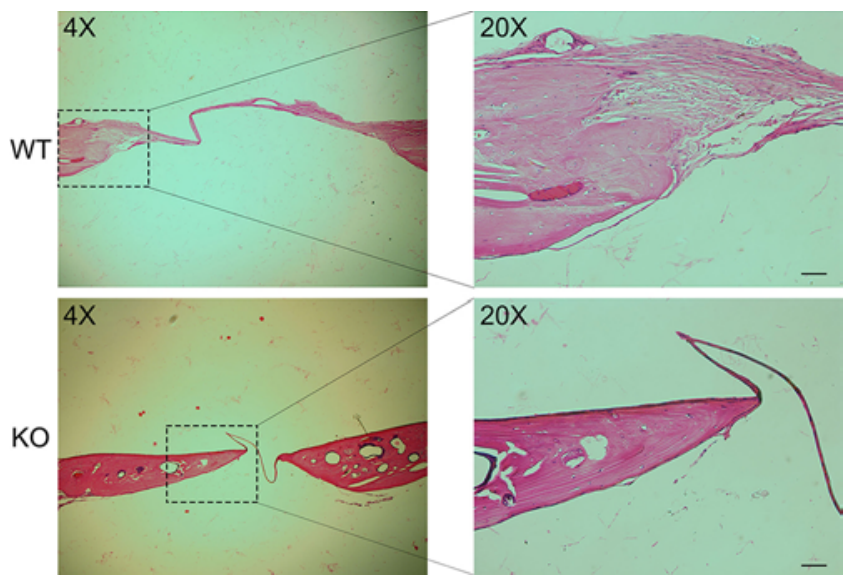

FIG. 2. Photomicrographs. New bone is seen on sagittal sections of calvarial defects. The dura was intact in both WT and Sost KO mice. An unhealed bone defect can be seen in both groups, but Sost KO mice had a shorter defect than WT mice. In addition, the morphology of the bone edge was different, with relatively sharp cutting edges of defects in WT mice with fibrous tissue, whereas gradual bone growth was found around the edge of defect in Sost KO mice, resulting in lamellar bone and little fibrous tissue. $\mathrm{H} \& \mathrm{E}$, scale bar $=200 \mu \mathrm{m}$. Figure is available in color online only.

mice was highly organized lamellar bone, which strongly indicates that bone regeneration originated from the edge of the defects. There was no indication of an inflammatory reaction at the defect site in either group.

\section{Wnt Signaling Pathway Involvement in Nonloaded Bone Regeneration}

To investigate whether the greater bone formation was obtained via an anabolic effect of Wnt signaling enhanced by the absence of sclerostin or whether another alternative signaling pathway was involved, intracellular signaling proteins were assayed from both the Wnt and BMP pathways. $\beta$-catenin expression was considerably upregulated in areas of active bone formation in Sost KO mice, while significantly decreased expression was observed in WT mice (Fig. 3). These areas of $\beta$-catenin expression overlapped with areas of differential expression of Sost between WT and KO mice, confirming earlier Western blot results (Fig. 1A). Conversely, we did not observe any remarkable differences between WT and Sost KO mice in expression of the representative marker (Smad 1/5/8 and Smad 2/3; Fig. 4). These data indicate that Sost KO mice exhibited highly activated Wnt signaling in the absence of sclerostin, compared with WT mice. The deletion of Sost did not provoke alternative BMP signaling-based bone regeneration in the defect site even as it enhanced the Wnt signaling, leading to significant new bone around the defect edges.

\section{Discussion}

Clinical management of bony healing differs depending on whether the craniofacial skeleton or the rest of the body is affected. In the long bones, rigid fixation and early weight bearing can lead to excellent outcomes. Loading is a known stimulus of bone deposition, with the negative regulator sclerostin directly associated with transduction of mechanical stimuli into a bony response. ${ }^{20,26}$ However, loading is clinically difficult for treatment of defects in the craniofacial skeleton, and bone healing occurs due to vascularized bone, or placement of bone flaps or grafts into a highly vascular bed. . $^{510,27}$

Although loading is not possible in the craniofacial skeleton, several lines of inquiry demonstrate that the same mechanisms that act on the weight-bearing skeleton also play a role above the clavicles. Mutations in sclerostin in humans are responsible for 2 known congenital diseases: sclerosteosis and van Buchem's disease. Both of these diseases are characterized by an increase in bone mass and a lower propensity for bony fracture. Interestingly, sclerosteosis has more severe manifestations characterized by cranial nerve foraminal stenosis, leading to blindness, deafness, and facial nerve palsies. ${ }^{8}$ In addition, mice that carry these mutations also have thicker cranial bone, and humans have increased intracranial pressure resulting from endocortical bone overgrowth. ${ }^{12,21}$ These converging pieces of data demonstrate that sclerostin-associated mechanotransduction is active in the craniofacial skeleton. Interestingly, sclerostin may have a more profound effect in the craniofacial skeleton because it is completely unloaded (i.e., sclerostin is maximally expressed), and removal of sclerostin may provide a greater enhancement of bone formation than in the loaded skeleton.

The results of this study demonstrate for the first time the effects of sclerostin in the nonloaded cranial skeleton. The purpose of this study was to determine the potential of a bone to repair itself while under the biochemical cues that would lead it to act as if it were under maximal loading conditions. It was demonstrated that even in a critical size calvarial defect (one that should not heal over the lifetime of the animal) bone formation would occur. The new bone formation was at the periphery, with osteocytes being the primary cell type to have created-ossified bone. Our results are consistent with those of previous studies demonstrating that decreased expression of sclerostin improves bone regeneration in rodent periodontitis models. ${ }^{25,33}$ Although these periodontitis models are within the craniofacial skeleton, they involve occlusal loading and thus do not completely mimic the nonloaded conditions present within the presently used cranial model.

Other features of the regenerated bone are worth noting. The dura was not a significant contributor to bone formation, as is expected in pediatric defects. Even though the suture mesenchymal stem cells appear to contribute to bone modeling and remodeling by giving rise to osteogenic fronts, periosteum, and/or dura, our micro-CT shows that the defected bone started being covered from the osteogenic front of the defect, rather than from periosteum and/or dura..$^{39}$ Furthermore, the regenerated bone in the Sost KO mice was lamellar bone (Fig. 2), which is evidence of outgrowth of new bone from the edge of the defect. When compared to other studies of calvarial defect repair, this study is one of the few that requires no scaffold for regeneration, and thus the relative contributions of the periosteum, dura, and osteogenic front in regeneration are more clearly demonstrated. ${ }^{14,16}$ Bone-specific $\beta$-catenin protein was most expressed at the advancing osteogenic fronts, while phosphor-Smads $1 / 5 / 8$ and $2 / 3$ in the BMP 


\section{$\beta$-catenin}

\section{Sost}

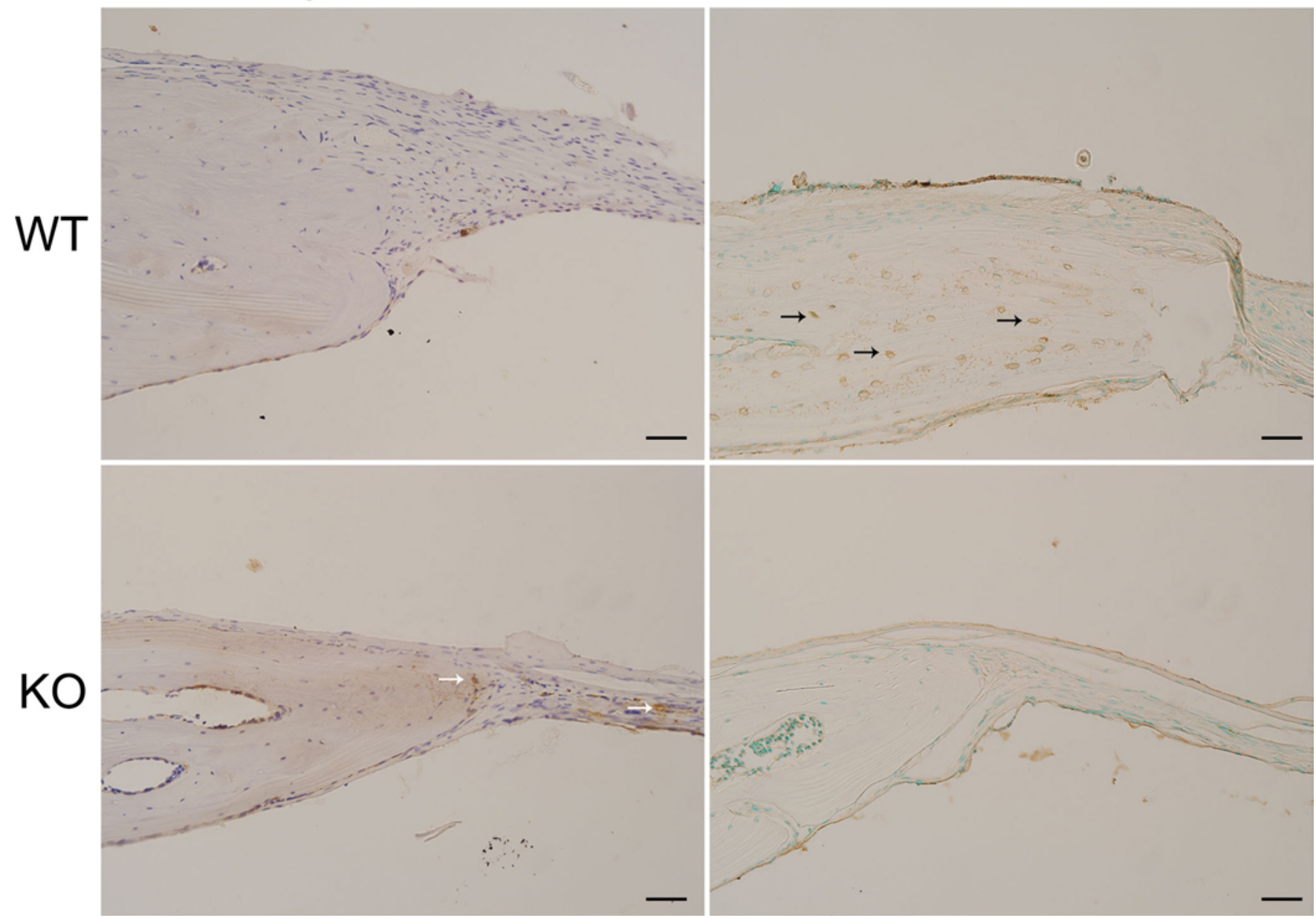

FIG. 3. Images of immunohistochemical analysis showing new bone formation via elevated Wnt signaling in Sost KO mice.

Compared with WT mice, higher levels of $\beta$-catenin were expressed in bone defects of Sost KO mice. White arrows indicate the detected $\beta$-catenin; note that $\beta$-catenin is expressed at the osteogenic front. In addition, we confirmed that sclerostin was highly expressed in osteocytes of WT mice, whereas we did not observe significant sclerostin expression in osteocytes in Sost KO mice. Black arrows indicate the detected sclerostin. Scale bar $=200 \mu \mathrm{m}$. Figure is available in color online only.

signaling pathway were not. Sclerostin does not appear to affect BMP signaling, but rather Wnt signaling in osteoblastic cells. ${ }^{34}$ Prior research has demonstrated that BMP signaling through the Type $1 \mathrm{~A}$ receptor (BMPR1A) in osteoblasts can regulate bone mass indirectly by inducing sclerostin-mediated downregulation of canonical Wnt signaling. ${ }^{11}$ Given this relationship between sclerostin and BMP signaling, directed investigation into the presence of BMP-induced bone formation showed that BMP signaling through Smads did not appear to contribute largely to new bone formation in Sost $\mathrm{KO}$ mice. Moreover, this BMP-independent new bone was laid down in an organized fashion in Sost KO mice, indicating the possibility of structurally sound bone.

\section{Conclusions}

This study demonstrates that bone healing in the nonweight-bearing skeleton can be accelerated with sclerostin downregulation. The finding that the mechanosensi- tive Wnt signaling pathway can be modulated in a relatively mechano-insensitive location opens a new avenue for the repair and regeneration of non-weight-bearing bones. Sclerostin inhibition has the potential to help heal bony defects in locations or situations, even in cases in which bony loading is an unfavorable choice. Currently, sclerostin inhibitory antibodies are in clinical testing for osteoporosis. There have been marked positive responses with minimal consequences with global inhibition. ${ }^{24}$ This study demonstrates that the removal of sclerostin function is useful in bone healing (and not just increases in bone density) and thus provides a foundation on which to test the new generation of sclerostin-inhibiting antibodies in an effort to further address the difficulties encountered by the practitioner treating these large bony defects.

\section{Acknowledgments}

This work was supported by NIH grant numbers AR53237 and BX001478 (to A.G.R.) and the Indiana Institute for Medical Research 2016 Young Investigator Award (to K.S.K.). 


\section{Smad $1 / 5 / 8$}

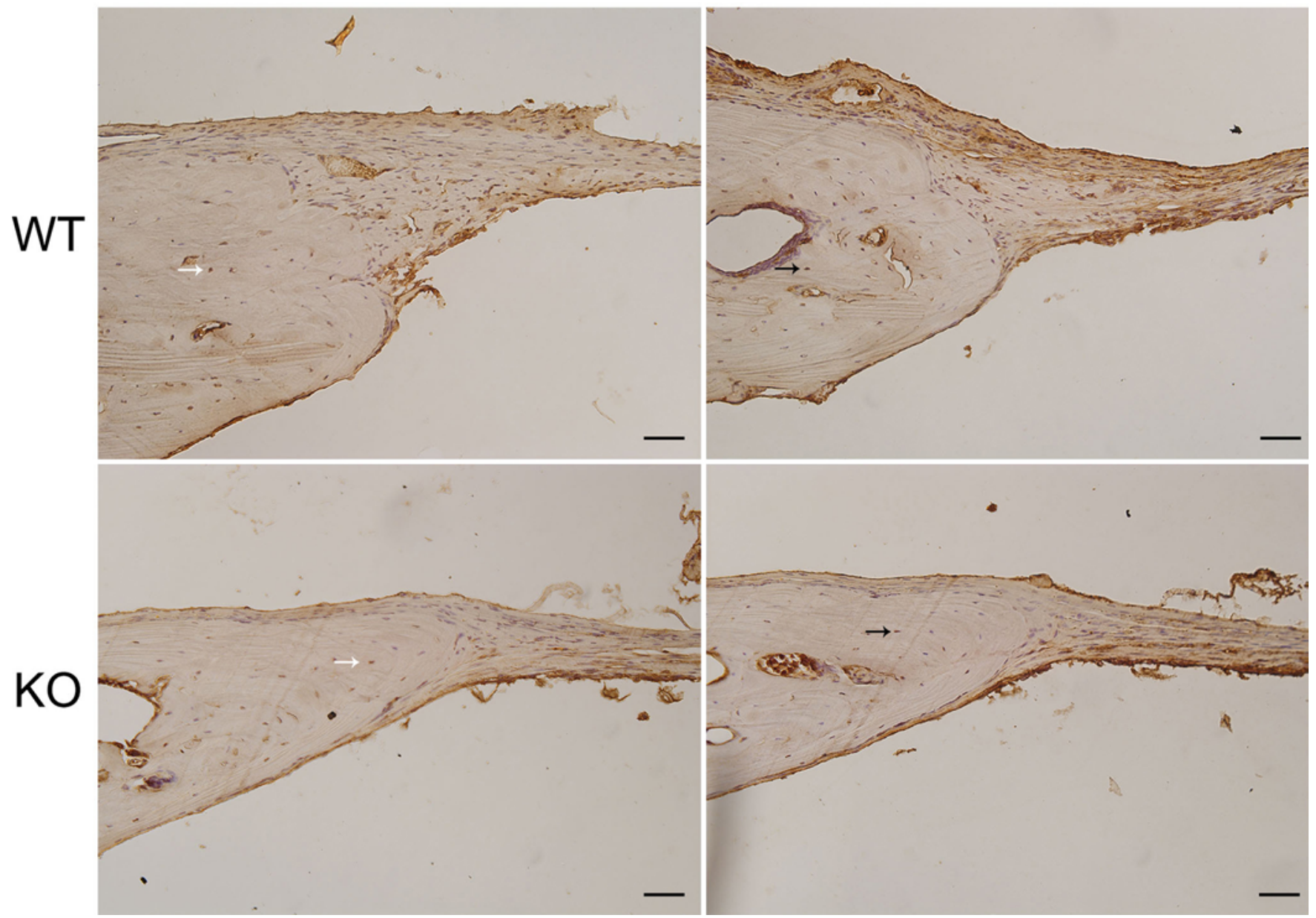

FIG. 4. Immunohistochemical staining for Smad1/5/8 and Smad 2/3 reveals no significant differences between WT and Sost KO mice. Deletion of Sost did not affect the BMP signaling pathway. White and black arrows indicate the detected Smad 1/5/8 and Smad $2 / 3$, respectively. Scale bar $=200 \mu \mathrm{m}$. Figure is available in color online only.

\section{References}

1. Bos GD, Goldberg VM, Zika JM, Heiple KG, Powell AE: Immune responses of rats to frozen bone allografts. J Bone Joint Surg Am 65:239-246, 1983

2. Connolly JF, Hahn H, Davy D: Fracture healing in weightbearing and nonweight-bearing bones. J Trauma 18:766770,1978

3. Cowan CM, Shi YY, Aalami OO, Chou YF, Mari C, Thomas $\mathrm{R}$, et al: Adipose-derived adult stromal cells heal critical-size mouse calvarial defects. Nat Biotechnol 22:560-567, 2004

4. Drosos GI, Touzopoulos P, Ververidis A, Tilkeridis K, Kazakos K: Use of demineralized bone matrix in the extremities. World J Orthop 6:269-277, 2015

5. Dwek JR: The periosteum: what is it, where is it, and what mimics it in its absence? Skeletal Radiol 39:319-323, 2010

6. Elsalanty ME, Genecov DG: Bone grafts in craniofacial surgery. Craniomaxillofac Trauma Reconstr 2:125-134, 2009

7. Gómez-Barrena E, Rosset P, Lozano D, Stanovici J, Ermthaller C, Gerbhard F: Bone fracture healing: cell therapy in delayed unions and nonunions. Bone 70:93-101, 2015

8. Hamersma H, Gardner J, Beighton P: The natural history of sclerosteosis. Clin Genet 63:192-197, 2003

9. Horowitz MC, Friedlaender GE: Immunologic aspects of bone transplantation. A rationale for future studies. Orthop Clin North Am 18:227-233, 1987

10. Huang C, Ness VP, Yang X, Chen H, Luo J, Brown EB, et al: Spatiotemporal analyses of osteogenesis and angiogenesis via intravital imaging in cranial bone defect repair. J Bone Miner Res 30:1217-1230, 2015

11. Kamiya N, Ye L, Kobayashi T, Mochida Y, Yamauchi M, Kronenberg HM, et al: BMP signaling negatively regulates bone mass through sclerostin by inhibiting the canonical Wnt pathway. Development 135:3801-3811, 2008

12. Keller H, Kneissel M: SOST is a target gene for PTH in bone. Bone 37:148-158, 2005

13. Krause C, Korchynskyi O, de Rooij K, Weidauer SE, de Gorter DJ, van Bezooijen RL, et al: Distinct modes of inhibition by sclerostin on bone morphogenetic protein and Wnt signaling pathways. J Biol Chem 285:41614-41626, 2010

14. Lee JW, Kang KS, Lee SH, Kim JY, Lee BK, Cho DW: Bone regeneration using a microstereolithography-produced customized poly(propylene fumarate)/diethyl fumarate photopolymer 3D scaffold incorporating BMP-2 loaded PLGA microspheres. Biomaterials 32:744-752, 2011

15. Levi B, Hyun JS, Montoro DT, Lo DD, Chan CK, Hu S, et al: In vivo directed differentiation of pluripotent stem cells for 
skeletal regeneration. Proc Natl Acad Sci U S A 109:20379_ 20384, 2012

16. Liu W, Kang N, Dong Y, Guo Y, Zhao D, Zhang S, et al: Effect of resorbable collagen plug on bone regeneration in rat critical-size defect model. Implant Dent 25:163-170, 2016

17. MacNabb C, Patton D, Hayes JS: Sclerostin antibody therapy for the treatment of osteoporosis: clinical prospects and challenges. J Osteoporos 2016:6217286, 2016

18. Moester MJC, Papapoulos SE, Löwik CWGM, van Bezooijen RL: Sclerostin: current knowledge and future perspectives. Calcif Tissue Int 87:99-107, 2010

19. Morse A, McDonald MM, Kelly NH, Melville KM, Schindeler A, Kramer I, et al: Mechanical load increases in bone formation via a sclerostin-independent pathway. J Bone Miner Res 29:2456-2467, 2014

20. Nguyen J, Tang SY, Nguyen D, Alliston T: Load regulates bone formation and sclerostin expression through a TGF $\beta$ dependent mechanism. PLoS One 8:e53813, 2013

21. Niziolek PJ, Farmer TL, Cui Y, Turner CH, Warman ML, Robling AG: High-bone-mass-producing mutations in the Wnt signaling pathway result in distinct skeletal phenotypes. Bone 49:1010-1019, 2011

22. Pagni G, Kaigler D, Rasperini G, Avila-Ortiz G, Bartel R, Giannobile WV: Bone repair cells for craniofacial regeneration. Adv Drug Deliv Rev 64:1310-1319, 2012

23. Pryor LS, Gage E, Langevin CJ, Herrera F, Breithaupt AD, Gordon CR, et al: Review of bone substitutes. Craniomaxillofac Trauma Reconstr 2:151-160, 2009

24. Recker RR, Benson CT, Matsumoto T, Bolognese MA, Robins DA, Alam J, et al: A randomized, double-blind phase 2 clinical trial of blosozumab, a sclerostin antibody, in postmenopausal women with low bone mineral density. J Bone Miner Res 30:216-224, 2015

25. Ren Y, Han X, Ho SP, Harris SE, Cao Z, Economides AN, et al: Removal of SOST or blocking its product sclerostin rescues defects in the periodontitis mouse model. FASEB J 29:2702-2711, 2015

26. Robling AG, Niziolek PJ, Baldridge LA, Condon KW, Allen MR, Alam I, et al: Mechanical stimulation of bone in vivo reduces osteocyte expression of Sost/sclerostin. J Biol Chem 283:5866-5875, 2008

27. Sadeghi M, Bakhshandeh B, Dehghan MM, Mehrnia MR, Khojasteh A: Functional synergy of anti-mir221 and nanohydroxyapatite scaffold in bone tissue engineering of rat skull. J Mater Sci Mater Med 27:132, 2016

28. Semënov M, Tamai K, He X: SOST is a ligand for LRP5/ LRP6 and a Wnt signaling inhibitor. J Biol Chem 280:26770-26775, 2005

29. Skerry TM: The response of bone to mechanical loading and disuse: fundamental principles and influences on osteoblast/ osteocyte homeostasis. Arch Biochem Biophys 473:117-123, 2008

30. Spatz JM, Ellman R, Cloutier AM, Louis L, van Vliet M, Suva LJ, et al: Sclerostin antibody inhibits skeletal deteriora- tion due to reduced mechanical loading. J Bone Miner Res 28:865-874, 2013

31. Spicer PP, Kretlow JD, Young S, Jansen JA, Kasper FK, Mikos AG: Evaluation of bone regeneration using the rat critical size calvarial defect. Nat Protoc 7:1918-1929, 2012

32. Suen PK, Qin L: Sclerostin, an emerging therapeutic target for treating osteoporosis and osteoporotic fracture: A general review. J Orthop Transl 4:13, 2016

33. Taut AD, Jin Q, Chung JH, Galindo-Moreno P, Yi ES, Sugai JV, et al: Sclerostin antibody stimulates bone regeneration after experimental periodontitis. J Bone Miner Res 28:2347-2356, 2013

34. van Bezooijen RL, Svensson JP, Eefting D, Visser A, van der Horst G, Karperien M, et al: Wnt but not BMP signaling is involved in the inhibitory action of sclerostin on BMP-stimulated bone formation. J Bone Miner Res 22:19-28, 2007

35. Wang T, He J, Zhang Y, Shi W, Dong J, Pei M, et al: A selective cell population from dermis strengthens bone regeneration. Stem Cells Transl Med [epub ahead of print], 2016

36. Ye JH, Xu YJ, Gao J, Yan SG, Zhao J, Tu Q, et al: Criticalsize calvarial bone defects healing in a mouse model with silk scaffolds and SATB2-modified iPSCs. Biomaterials 32:5065-5076, 2011

37. Yee CS, Xie L, Hatsell S, Hum N, Murugesh D, Economides AN, et al: Sclerostin antibody treatment improves fracture outcomes in a Type I diabetic mouse model. Bone 82:122134, 2016

38. Zhao H, Feng J, Ho TV, Grimes W, Urata M, Chai Y: The suture provides a niche for mesenchymal stem cells of craniofacial bones. Nat Cell Biol 17:386-396, 2015

39. Zuk PA: Tissue engineering craniofacial defects with adult stem cells? Are we ready yet? Pediatr Res 63:478-486, 2008

\section{Disclosures}

The authors report no conflict of interest concerning the materials or methods used in this study or the findings specified in this paper.

\section{Author Contributions}

Conception and design: Tholpady, Robling. Acquisition of data: Kang, Lastfogel. Analysis and interpretation of data: Tholpady, Kang. Drafting the article: Kang. Critically revising the article: Tholpady. Reviewed submitted version of manuscript: Tholpady. Approved the final version of the manuscript on behalf of all authors: Tholpady. Administrative/technical/material support: Tholpady, Ackerman, Jea, Robling. Study supervision: Tholpady, Kang.

\section{Correspondence}

Sunil S. Tholpady, Department of Surgery, Indiana University School of Medicine, 705 Riley Hospital Dr., RI 2514, Indianapolis, IN 46202.email: stholpad@iupui.edu. 